\title{
DESARROLLO PRODUCTIVO Y CUALITATIVO DE MAÍZ HÍBRIDO PARA ENSILAJE ${ }^{1}$
}

\author{
Carlos Boschini², Jorge Elizondo ${ }^{2}$
}

\begin{abstract}
RESUMEN
Desarrollo productivo y cualitativo de maíz híbrido para ensilaje. El experimento se llevó a cabo en la Estación Experimental "Alfredo Volio Mata" de la Universidad de Costa Rica, en agosto del 2002. Se estudió el crecimiento y la calidad bromatológica del maíz híbrido $3002 \mathrm{~W}$ blanco (Pionner), $90 \%$ de germinación, recomendado para la zona alta de la provincia de Cartago. Se determinó la producción y contenido de materia seca por hectárea, proteína cruda, las cenizas, la fibra neutro detergente y las fracciones que la componen y la energía digestible, desde 70 hasta los 154 días de crecimiento. Los contenidos de proteína cruda y energía digestible fueron altos hasta los 84 días ( $>12 \%$ PC y $>2,8 \mathrm{mcal} / \mathrm{kg}$ ED) y medios hasta los 112 días ( $>9 \%$ PC y $>2,3 \mathrm{mcal} / \mathrm{kg}$ ED), con contenidos de materia seca inferiores a $15 \%$, aún no recomendado para ensilaje. A los 126 días alcanzó un rendimiento de 17,9 t/ha, con $20 \%$ de materia seca, $7,9 \%$ de proteína cruda, $2,2 \mathrm{mcal} \mathrm{ED} / \mathrm{kg}, 76 \%$ de fibra neutro detergente y $4,8 \%$ de lignina.
\end{abstract}

\begin{abstract}
Productive and qualitative development of corn hybrid for ensiling. The experiment was carried out at the Experimental Station "Alfredo Volio Mata" of the University of Costa Rica. It is located to an altitude of $1542 \mathrm{~m}$. We evaluated growth and quality of the white corn hybrid $3002 \mathrm{~W}$ (Pioneer) recommended for the high elevation areas of the province of Cartago, Costa Rica. Dry matter yield per hectare, dry matter content, crude protein, ashes, neutral detergent fiber and its fractions as well as digestible energy were determined from 70 to 154 days of growth. Corn ears started to appear at day 84 . Crude protein and digestible energy were high until day 84 (>12\% CP and >2,8 Mcal DE/kg DM) and became lower after day 112 ( $>9 \% \mathrm{CP}$ and $>2,3 \mathrm{Mcal} \mathrm{DE} / \mathrm{kg}$ $\mathrm{DM}$ ), with less than $15 \%$ dry matter which is not recommended for ensiling. At 126 days of growth it yielded 17,9 $\mathrm{t}$ of dry matter per hectare with $20 \%$ dry matter content, $7,9 \%$ crude protein, 2,2 Mcal ED/kg DM, 76\% neutral detergent fiber and $4,8 \%$ lignin.
\end{abstract}

\section{INTRODUCCIÓN}

La necesidad de atender la demanda de nutrientes en el ganado lechero, de forma constante a través del año, se inicia con un balance forrajero, fundamentado en las metas de productividad animal de la finca, el crecimiento y la disponibilidad estacional de los forrajes y el almacenamiento de una reserva forrajera para disponer oportunamente. El forraje de maíz ha sido empleado con mucha frecuencia en los países subtropicales como parte del balance forrajero anual (Bruno et al. 1995, Cuomo et al. 1998), formando la reserva generalmente ensilada (Aldrich y Leng 1974). En Costa Rica, la práctica de ensilaje ha tomado auge en los último seis años (Boschini 2002), obteniendo experiencia los productores en los aspectos metodológicos del proceso. El cultivo de maíz ha sido tradicional para la producción de grano en Costa Rica, especialmente en las zonas bajas. No se cuenta con la misma experiencia en las zonas lecheras de altura, en cuanto a variedades e híbridos, densidades de siembra y edades de cosecha óptima para la producción de forraje. Materiales genéticos criollos de la parte alta de Cartago han dado rendimientos de 11,2 a 15,1 t/ha de materia seca (107 días) con relaciones:

1 Recibido para publicación el 22 de octubre de 2003. Inscrito en la Vicerrectoría de Investigación, Proyecto No. 737-A2-131.

2 Estación Experimental Alfredo Volio Mata. Facultad de Ciencias Agroalimentarias. Universidad de Costa Rica. Cartago, Costa Rica. 
tallo de 0,53 usando la variedad amarilla (Amador y Boschini 2000). En similares condiciones, el criollo blanco registró rendimientos de 15 a 20 t/ha de materia seca (147 días) y el híbrido $3002 \mathrm{~W} ®$ de 7 a 9,2 t/ha (119 días), con relaciones hoja:tallo de 0,62 y 0,34 respectivamente (Boschini y Elizondo 2004).

Es evidente que las variedades criollas, adaptadas a alturas superiores de $1.500 \mathrm{msnm}$, muestran rendimientos de forraje altos, con degradabilidades ruminales de la materia seca en el orden de 77 y $74 \%$ a los 121 y 135 días, respectivamente (Boschini y Amador 2001). El empleo masivo de las variedades criollas está limitado por la disponibilidad de semilla y la debida certificación. Por ambas razones, la siembra de materiales híbridos para la producción de forraje en terrenos altos se hace cada vez más frecuente. Esta investigación se llevó a cabo con el propósito de estudiar el rendimiento y la concentración de nutrientes en el maíz híbrido a través del período de crecimiento y determinar el estado óptimo de cosecha para ensilar.

\section{MATERIALES Y MÉTODOS}

\section{Localización y siembra}

El experimento se llevó a cabo en la Estación Experimental "Alfredo Volio Mata" de la Universidad de Costa Rica, ubicada en Ochomogo de Cartago a 1.542 msnm, con una precipitación media anual de $2.050 \mathrm{~mm}$, distribuida en los meses de mayo a noviembre. La temperatura media es de $19,5^{\circ} \mathrm{C}$ y la humedad relativa media es de $84 \%$. El suelo es de origen volcánico, clasificado como Typic Distrandepts (Vásquez 1982). Se caracteriza por una profundidad media, con buen drenaje natural y una fertilidad media. El terreno empleado tiene un $\mathrm{pH}$ de 6,0. La zona se tipifica ecológicamente como bosque húmedo montano bajo (Tosi 1970, citado por Vásquez 1982).

Se aró y rastreó una hectárea de terreno. Se fertilizó con $368 \mathrm{~kg}$ de la fórmula 10-30-10. Se repitió la rastrea y se rayó a $70 \mathrm{~cm}$ entre surcos. La siembra se hizo el 6 de agosto del 2002 con 25 kg de semilla de maíz híbrido $3002 \mathrm{~W} 囚$ blanco (Pionner), 90\% de germinación, recomendado para la zona alta de la provincia de Cartago, distribuida a $50 \mathrm{~cm}$ entre plantas en forma manual, depositando dos semillas por golpe (equivalente a 58.000 plantas/ha). Dos días después, se aplicó un herbicida preemergente y posemergente (Atrazina 900 $\mathrm{g} / \mathrm{kg}$ ) disuelto en 200 litros de agua y asperjado con bomba de espalda. A los 42 días de la siembra se aplicó $45,5 \mathrm{~kg}$ de nitrógeno en forma de nitrato de amonio.

\section{Diseño experimental y muestreo}

Se usó un diseño de bloques completos al azar (Steel y Torrie 1988.) con cuatro repeticiones y seis tratamientos: 70, 84, 98, 112, 126 y 154 días de crecimiento. El terreno fue dividido en 4 lotes iguales de 2.500 $\mathrm{m}^{2}$. Cada uno fue dividido en seis unidades experimentales donde se distribuyeron aleatoriamente las edades de cosecha. Los sitios de muestreos dentro de cada unidad experimental se seleccionaron al azar, recolectando 10 metros lineales en el surco más cercano, medido a partir del punto medio entre dos golpes de siembra consecutivos, las plantas fueron cortadas a $10 \mathrm{~cm}$ sobre el nivel de suelo. Esta operación se repitió en los dos surcos aledaños. Las plantas cosechadas fueron pesadas para estimar la producción de biomasa, separando previamente los tallos, hojas y mazorcas. Cada parte de la planta fue cortada en trozos de $2 \mathrm{~cm}$ de largo con una picadora industrial. El material fue homogenizado con herramientas de campo, se extrajo una muestra de $10 \mathrm{~kg}$ para el laboratorio, donde fueron pesadas y secadas en un horno a $60{ }^{\circ} \mathrm{C}$ durante 48 horas, hasta alcanzar un peso constante, y molidos con un tamiz de $1 \mathrm{~mm}$.

\section{Análisis de laboratorio}

Se determinó el contenido de materia seca en una estufa a $105^{\circ} \mathrm{C}$, la proteína cruda por el método Kjeldahl y la cenizas totales (AOAC 2000). La fibra neutro detergente, la fibra ácido detergente y la lignina fueron analizadas por el método de Goering y Van Soest (1970). Los valores de hemicelulosa y celulosa se estimaron por diferencia de las fracciones correspondientes. La energía digestible se determinó a partir de la digestibilidad in vitro de la materia seca (Tilley y Terry 1963).

\section{Variables en estudio y análisis estadístico}

Se estimó la producción de materia verde (MV) por hectárea y fue determinada la concentración de materia seca (MS), proteína cruda (PC), fibra neutro detergente (FND) y sus componentes: fibra ácido detergente (FAD), hemicelulosa (HEMI), celulosa (CEL) y lignina (LIG); las cenizas (CEN) y la energía digestible (ED), a partir de la digestibilidad in vitro (DIVMS), tanto en hojas como en tallos, mazorcas y en la planta completa. Se cuantificó el rendimiento por hectárea de cada componente bromatológico en cada una de las edades de crecimiento. Las variables fueron sometida al análisis de varianza con el PROC ANOVA del paquete estadístico SAS (1985). Aquellas que mostraron diferencias importantes entre medias, fueron sometidas a la prueba de Duncan para su separación estadística. 


\section{RESULTADOS}

\section{Composición química}

El contenido de materia seca en la planta varió $(\mathrm{P}<0,01)$ a través del período de crecimiento, como se observa en el Cuadro 1. La concentración fue mayor en las hojas que en los tallos en todas las edades, mostrándose a partir de los 70 días de crecimiento un incremento sostenido hasta los 112 días, para luego mantenerse constante entre 21 y $22 \%$. En el tallo se manifestó un crecimiento en la concentración de materia seca con incrementos decrecientes. Las mazorcas aparecieron entre los 84 y 98 días, con un incremento sostenido en el contenido de materia seca a partir de los 112 días. Los valores en la planta entera (Figura 1) constituyen un valor medio ponderado por la masa producida en cada parte de la planta. Los contenidos de proteína cruda fueron altos a los 70 días y disminuyeron $(\mathrm{P}<0,01)$ conforme aumentó la edad de la planta, observándose que la mayor concentración está en las hojas, luego en mazorca y tallo. Similar respuesta se observó en la diges- tibilidad y la concentración de energía digestible conforme avanzó el crecimiento. El contenido de fibra

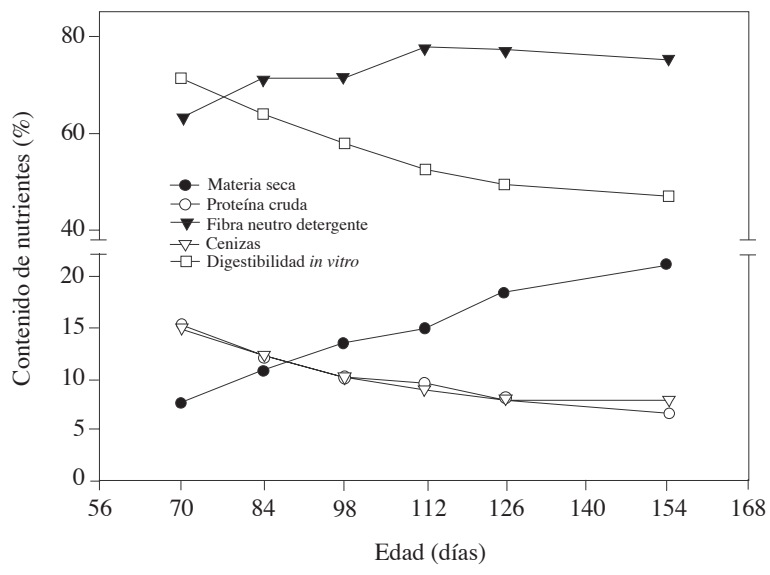

Figura 1. Contenidos de materia seca y composición nutricional de la planta entera de maíz en diferentes edades de crecimiento. Tres Ríos, Cartago, 2003.

Cuadro 1. Contenido bromatológico de la planta de maíz y en las partes que la conforman, a través del periodo de crecimiento. Tres Ríos, Cartago, 2003.

\begin{tabular}{|c|c|c|c|c|c|c|c|c|c|c|c|}
\hline $\begin{array}{l}\text { Parte } \\
\text { planta }\end{array}$ & $\begin{array}{l}\text { Edad } \\
\text { días }\end{array}$ & $\begin{array}{c}\text { MS }^{1} \\
\%\end{array}$ & $\begin{array}{c}\text { PC }^{1} \\
\%\end{array}$ & $\begin{array}{c}\text { FND }^{1} \\
\%\end{array}$ & $\begin{array}{c}\text { FAD }^{1} \\
\%\end{array}$ & $\begin{array}{c}\text { HEMI }^{1} \\
\%\end{array}$ & $\begin{array}{c}\text { CEL }^{1} \\
\%\end{array}$ & $\begin{array}{c}\text { LIG }^{1} \\
\%\end{array}$ & $\begin{array}{c}\text { CEN1 }^{1} \\
\%\end{array}$ & $\begin{array}{c}\text { DIVMS }^{1} \\
\%\end{array}$ & $\begin{array}{c}\mathrm{ED}^{1,2} \\
\mathrm{mcal} / \mathrm{kg}\end{array}$ \\
\hline \multirow[t]{6}{*}{ Hoja } & 70 & $10.56 a^{*}$ & $16,75 \mathrm{~d}$ & $64,42 \mathrm{a}$ & $39,79 \mathrm{a}$ & $24,63 \mathrm{a}$ & $35,52 \mathrm{a}$ & $4,27 \mathrm{a}$ & 13,52 & $71,06 \mathrm{c}$ & $3,13 \mathrm{c}$ \\
\hline & 84 & $13,99 \mathrm{~b}$ & $15,42 \mathrm{~d}$ & $71,14 \mathrm{~b}$ & $40,06 \mathrm{a}$ & $31,08 \mathrm{~b}$ & $35,73 \mathrm{a}$ & $4,33 \mathrm{a}$ & 13,55 & $64,37 \mathrm{~b}$ & $2,84 \mathrm{~b}$ \\
\hline & 98 & $17,54 \mathrm{~b}$ & $13,36 \mathrm{c}$ & $73,19 \mathrm{~b}$ & $40,53 \mathrm{a}$ & $32,66 \mathrm{~b}$ & $36,29 \mathrm{a}$ & $4,24 \mathrm{a}$ & 13,59 & $61,64 \mathrm{~b}$ & $2,72 \mathrm{~b}$ \\
\hline & 112 & $21,09 \mathrm{c}$ & $11,88 \mathrm{bc}$ & $77,61 \mathrm{c}$ & $42,03 \mathrm{~b}$ & $35,58 \mathrm{c}$ & $37,41 \mathrm{ab}$ & $4,62 \mathrm{a}$ & 13,63 & $55,13 \mathrm{ab}$ & $2,43 \mathrm{ab}$ \\
\hline & 126 & $21,66 \mathrm{c}$ & $10,41 \mathrm{~b}$ & $77,93 \mathrm{c}$ & $43,08 \mathrm{~b}$ & $34,85 \mathrm{c}$ & $37,94 \mathrm{ab}$ & $5,14 a b$ & 13,69 & $47,61 \mathrm{a}$ & $2,10 \mathrm{a}$ \\
\hline & 154 & $21,39 \mathrm{c}$ & $8,23 \mathrm{a}$ & $78,95 \mathrm{c}$ & $47,03 \mathrm{c}$ & $31,92 \mathrm{~b}$ & $39,84 \mathrm{~b}$ & $7,19 \mathrm{~b}$ & 13,76 & $43,59 \mathrm{a}$ & $1,92 \mathrm{a}$ \\
\hline \multirow[t]{6}{*}{ Tallo } & 70 & $5,45 \mathrm{a}$ & $12,08 \mathrm{e}$ & $60,87 \mathrm{a}$ & $39,57 \mathrm{a}$ & $21,30 \mathrm{a}$ & $36,42 \mathrm{a}$ & $3,15 \mathrm{a}$ & $16,11 \mathrm{~d}$ & $70,90 \mathrm{~d}$ & $3,13 \mathrm{~d}$ \\
\hline & 84 & $8,48 \mathrm{~b}$ & $8,21 \mathrm{~d}$ & $69,91 \mathrm{ab}$ & $44,71 \mathrm{ab}$ & $25,20 \mathrm{~b}$ & $40,89 \mathrm{~b}$ & $3,82 \mathrm{a}$ & $10,83 \mathrm{c}$ & $63,33 \mathrm{c}$ & $2,79 \mathrm{c}$ \\
\hline & 98 & $12,12 \mathrm{c}$ & $7,14 \mathrm{c}$ & $72,83 \mathrm{ab}$ & $46,75 \mathrm{c}$ & $26,08 \mathrm{~b}$ & $41,70 \mathrm{~b}$ & $5,05 \mathrm{ab}$ & $8,38 \mathrm{~b}$ & $62,50 \mathrm{c}$ & $2,76 \mathrm{c}$ \\
\hline & 112 & $13,91 \mathrm{~cd}$ & $5,93 \mathrm{~b}$ & $74,79 \mathrm{ab}$ & $46,72 \mathrm{c}$ & $28,07 \mathrm{c}$ & $40,75 \mathrm{~b}$ & $5,97 \mathrm{~b}$ & $6,74 \mathrm{a}$ & $50,21 \mathrm{~b}$ & $2,21 \mathrm{~b}$ \\
\hline & 126 & $15,02 \mathrm{~d}$ & $4,41 \mathrm{ab}$ & $76,08 \mathrm{~b}$ & $46,92 \mathrm{c}$ & $29,16 \mathrm{c}$ & $40,61 \mathrm{~b}$ & $6,31 \mathrm{~b}$ & $6,93 \mathrm{a}$ & $49,02 \mathrm{~b}$ & $2,16 \mathrm{~b}$ \\
\hline & 154 & $15,88 \mathrm{~d}$ & $4,12 \mathrm{a}$ & $77,71 \mathrm{~b}$ & $49,39 \mathrm{c}$ & $28,32 \mathrm{c}$ & $42,28 \mathrm{~b}$ & $7,11 \mathrm{~b}$ & $7,27 a b$ & $38,09 \mathrm{a}$ & $1,68 \mathrm{a}$ \\
\hline \multirow[t]{4}{*}{ Mazorca } & 98 & $9,09 \mathrm{a}$ & $13,04 \mathrm{~d}$ & $61,70 \mathrm{a}$ & $31,74 \mathrm{c}$ & 29,96 & $28,97 \mathrm{c}$ & 2,77 & $7,29 \mathrm{c}$ & $77,21 \mathrm{~b}$ & $3,40 \mathrm{~b}$ \\
\hline & 112 & $11,57 \mathrm{a}$ & $10,42 \mathrm{c}$ & $80,14 \mathrm{c}$ & $30,84 \mathrm{c}$ & 49,30 & $27,91 \mathrm{c}$ & 2,93 & $4,54 \mathrm{~b}$ & 63,35 & a 2,79 a \\
\hline & 126 & $18,87 \mathrm{~b}$ & $8,89 \mathrm{~b}$ & $76,49 \mathrm{~b}$ & $27,11 b$ & 49,38 & $24,03 \mathrm{~b}$ & 3,08 & $3,55 \mathrm{a}$ & 59,40 & a 2,62 a \\
\hline & 154 & $30,81 \mathrm{c}$ & $7,63 \mathrm{a}$ & $70,06 \mathrm{~b}$ & $22,29 \mathrm{a}$ & 47,77 & $19,74 \mathrm{a}$ & 2,55 & $3,42 \mathrm{a}$ & 57,74 & a 2,55 a \\
\hline \multirow[t]{6}{*}{ Entera } & 70 & $7,77 \mathrm{a}$ & $14,96 \mathrm{~d}$ & $63,06 \mathrm{a}$ & $39,71 \mathrm{a}$ & $23,35 \mathrm{a}$ & 35,87 & $3,84 \mathrm{a}$ & $14,51 \mathrm{c}$ & $71,04 \mathrm{e}$ & $3,13 \mathrm{e}$ \\
\hline & 84 & $10,73 \mathrm{~b}$ & $12,04 \mathrm{c}$ & $70,56 \mathrm{~b}$ & $42,24 \mathrm{~b}$ & $28,33 \mathrm{~b}$ & 38,15 & $4,09 \mathrm{a}$ & $12,28 \mathrm{c}$ & $64,06 \mathrm{~d}$ & $2,82 \mathrm{~d}$ \\
\hline & 98 & $13,25 \mathrm{c}$ & $10,20 \mathrm{~b}$ & $71,79 \mathrm{~b}$ & $42,73 \mathrm{~b}$ & $29,06 \mathrm{~b}$ & 38,24 & $4,49 \mathrm{~b}$ & $10,30 \mathrm{~b}$ & $58,25 \mathrm{c}$ & $2,57 \mathrm{c}$ \\
\hline & 112 & $14,99 \mathrm{~d}$ & $9,21 \mathrm{~b}$ & $77,16 \mathrm{c}$ & $41,02 \mathrm{ab}$ & $36,14 \mathrm{c}$ & 36,30 & $4,72 \mathrm{~b}$ & $8,67 \mathrm{ab}$ & 52,45 & b $2,31 \mathrm{~b}$ \\
\hline & 126 & $18,09 \mathrm{e}$ & $7,90 \mathrm{ab}$ & $76,81 \mathrm{c}$ & $38,71 \mathrm{a}$ & $38,11 \mathrm{c}$ & 33,91 & $4,80 \mathrm{~b}$ & $7,89 \mathrm{a}$ & $49,81 \mathrm{ab}$ & $2,20 \mathrm{ab}$ \\
\hline & 154 & $21,02 \mathrm{f}$ & $6,55 \mathrm{a}$ & $75,41 \mathrm{c}$ & $39,25 \mathrm{a}$ & $36,15 \mathrm{c}$ & 33,71 & $5,54 \mathrm{c}$ & $7,83 \mathrm{a}$ & $46,85 \mathrm{a}$ & $2,07 \mathrm{a}$ \\
\hline
\end{tabular}

1 MS materia seca, PC proteína cruda, FND fibra neutro detergente, FAD fibra ácida detergente, HEMI hemielulosa, CEL celulosa, LIG lignina, CEN cenizas, DIVMS digestibilidad in vitro de la materia seca, ED energía digestible.

$2 \mathrm{ED}=$ DIVMS $* 4,409 / 100$.

* Letras distintas marcan diferencia significativas $(\mathrm{P}<0,05)$. 
neutro detergente fue alto en todas las partes de la planta, aún cuando se encontraron diferencias de importancia $(\mathrm{P}<0,05)$ entre edades, particularmente a los 70 días. La concentración de fibra ácido detergente fue ligeramente superior en el tallo que en la hoja, mayor que en la mazorca. Los contenidos de hemicelulosa fueron mayores en la hoja que en el tallo. La concentración de lignina fue constante hasta los 112 días $(4,2-4,3 \%)$, para luego mostrar un incremento sostenido $(\mathrm{P}<0,05)$ hasta 7,2\% a los 154 días. En el tallo se observó una acumulación, cada vez mayor, conforme aumentó la edad de la planta, hasta alcanzar 7,1\% a los 154 días. La acumulación de lignina en la mazorca fue de 2,5 a 3,1\%. La concentración de cenizas en la hoja fue muy uniforme $(\mathrm{P}>0,05)$ a través del periodo de crecimiento, mientras que en el tallo y en la mazorca se mostró un pérdida $(\mathrm{P}<0,01)$ conforme aumentó la edad. En la planta entera se encontró un 14,5\% a los 70 días y 7,8 \% a los 154 días (Figura 1).

\section{Rendimiento por área}

La producción de materia verde por ha presentó diferencias $(\mathrm{P}<0,01)$ a través del periodo de crecimiento.
A los 70 días se estimó un rendimiento de 51,2 t/ha, creciendo a $111,4 \mathrm{t} / \mathrm{ha}$ a los 112 días y decayendo a 84.1 t/ha a los 154 días. A partir de los 70 días, la cantidad de materia fresca en la fracción de tallo fue siempre mayor que en la fracción de hoja (Cuadro 2). La producción total de materia seca mostró una curva de crecimiento $(\mathrm{P}<0,01)$ con un valor asintótico de 17.9 ton/ha a los 126 días (Figura 2), observándose una ligera pérdida de $274 \mathrm{~kg} / \mathrm{ha}$ a los 154 días. El mayor rendimiento de hoja se obtuvo a los 112 días, de tallo a los 98 días y de mazorca a lo 154 días de crecimiento. La pérdida de materia seca en hojas y tallos a partir de los 98-112 días es menor a la contribución de materia seca durante el crecimiento de la mazorca. En el Cuadro 3 se presenta la contribución porcentual de cada fracción de la planta (relación hoja:tallo:mazorca) al rendimiento de materia seca total. Se observa que la fracción de hojas disminuye desde los 70 días, y se incrementa la proporción de tallo hasta los 98 días, luego empieza a decrecer la contribución relativa en el rendimiento total de materia seca. Se observa que a partir de esa edad, comienza a participar la producción de mazorca en forma creciente hasta los 154 días (Figura 3). La proteína retenida en la materia seca total fue de 1,5 t/ha a los 112 días y la energía digestible de 41,3 gigacalorías/ha a los

Cuadro 2. Producción de materia verde, materia seca, proteína, fibra y energía de la planta de maíz y las partes componentes, a través del periodo de crecimiento. Tres Ríos, Cartago, 2003.

\begin{tabular}{|c|c|c|c|c|c|c|c|}
\hline $\begin{array}{l}\text { Parte } \\
\text { planta }\end{array}$ & $\begin{array}{c}\text { Edad } \\
\text { días }\end{array}$ & $\begin{array}{l}\text { MV1 } \\
\text { kg/ha }\end{array}$ & $\begin{array}{c}\text { MS1 } \\
\text { kg/ha }\end{array}$ & $\begin{array}{c}\text { PC1 } \\
\text { kg/ha }\end{array}$ & $\begin{array}{l}\text { FND1 } \\
\text { kg/ha }\end{array}$ & $\begin{array}{l}\text { CEN1 } \\
\text { kg/ha }\end{array}$ & $\begin{array}{c}\text { ED1 } \\
\text { mcal/ha }\end{array}$ \\
\hline \multirow[t]{6}{*}{ Hoja } & 70 & $23.228 \mathrm{a}$ & $2.453 \mathrm{a}$ & $411 \mathrm{a}$ & $1.580 \mathrm{a}$ & $332 \mathrm{a}$ & $7.685 \mathrm{a}$ \\
\hline & 84 & $28.714 \mathrm{~b}$ & $4.017 \mathrm{~b}$ & $619 b$ & $2.858 \mathrm{~b}$ & $544 \mathrm{~b}$ & $11.409 \mathrm{bc}$ \\
\hline & 98 & $31.429 \mathrm{c}$ & $5.513 \mathrm{c}$ & $736 \mathrm{c}$ & $4.035 \mathrm{c}$ & 749 bc & $14.994 \mathrm{c}$ \\
\hline & 112 & $28.572 \mathrm{~b}$ & $6.026 \mathrm{~d}$ & $716 \mathrm{c}$ & $4.677 \mathrm{c}$ & $821 \mathrm{c}$ & $14.643 \mathrm{c}$ \\
\hline & 126 & $26.429 \mathrm{ab}$ & $5.725 \mathrm{~cd}$ & $596 \mathrm{~b}$ & $4.461 \mathrm{c}$ & 784 bc & $12.016 \mathrm{bc}$ \\
\hline & 154 & $24.286 \mathrm{a}$ & $5.195 \mathrm{c}$ & $428 \mathrm{a}$ & $4.101 \mathrm{c}$ & $715 \mathrm{bc}$ & 9.984 b \\
\hline \multirow[t]{6}{*}{ Tallo } & 70 & $28.029 \mathrm{a}$ & $1.528 \mathrm{a}$ & $185 \mathrm{a}$ & $930 \mathrm{a}$ & $246 \mathrm{a}$ & $4.775 \mathrm{a}$ \\
\hline & 84 & $41.714 \mathrm{bc}$ & $3.537 \mathrm{~b}$ & $290 \mathrm{a}$ & $2.473 \mathrm{~b}$ & $383 \mathrm{ab}$ & $9.877 \mathrm{~b}$ \\
\hline & 98 & $58.571 \mathrm{c}$ & $7.099 \mathrm{c}$ & $507 \mathrm{c}$ & $5.170 \mathrm{c}$ & $595 \mathrm{c}$ & $19.562 \mathrm{c}$ \\
\hline & 112 & $46.429 \mathrm{bc}$ & $6.458 \mathrm{c}$ & $383 \mathrm{ab}$ & $4.830 \mathrm{c}$ & $435 \mathrm{~b}$ & $14.297 \mathrm{bc}$ \\
\hline & 126 & $39.286 \mathrm{~b}$ & $5.901 \mathrm{c}$ & $260 \mathrm{a}$ & $4.489 \mathrm{c}$ & $409 \mathrm{~b}$ & $12.753 \mathrm{bc}$ \\
\hline & 154 & $39.857 \mathrm{~b}$ & $6.329 \mathrm{c}$ & $261 \mathrm{a}$ & $4.918 \mathrm{c}$ & $460 \mathrm{~b}$ & $10.629 \mathrm{~b}$ \\
\hline \multirow[t]{4}{*}{ Mazorca } & 98 & $16.429 \mathrm{a}$ & $1.493 \mathrm{a}$ & $195 \mathrm{a}$ & $921 \mathrm{a}$ & 109 & $5.084 \mathrm{a}$ \\
\hline & 112 & $36.429 \mathrm{~b}$ & $4.215 \mathrm{ab}$ & $439 \mathrm{ab}$ & $3.378 \mathrm{ab}$ & 191 & $11.772 \mathrm{~b}$ \\
\hline & 126 & $33.571 \mathrm{~b}$ & $6.335 \mathrm{~b}$ & $563 \mathrm{~b}$ & $4.846 \mathrm{~b}$ & 225 & $16.591 \mathrm{c}$ \\
\hline & 154 & $20.000 \mathrm{a}$ & $6.162 \mathrm{~b}$ & $470 \mathrm{ab}$ & $4.317 \mathrm{ab}$ & 211 & $15.687 \mathrm{c}$ \\
\hline \multirow[t]{6}{*}{ Entera } & 70 & $51.257 \mathrm{a}$ & $3.980 \mathrm{a}$ & $595 \mathrm{a}$ & $2510 \mathrm{a}$ & $578 \mathrm{a}$ & $12.460 \mathrm{a}$ \\
\hline & 84 & $70.428 \mathrm{~b}$ & $7.554 \mathrm{~b}$ & $910 \mathrm{ab}$ & $5331 \mathrm{~b}$ & $927 \mathrm{a}$ & $21.286 \mathrm{~b}$ \\
\hline & 98 & $106.429 \mathrm{~d}$ & $14.105 \mathrm{c}$ & $1.438 \mathrm{~b}$ & $10.126 \mathrm{bc}$ & $1.453 \mathrm{~b}$ & $39.640 \mathrm{c}$ \\
\hline & 112 & $111.430 \mathrm{~d}$ & $16.699 \mathrm{c}$ & $1.538 \mathrm{~b}$ & $12.885 \mathrm{c}$ & $1.448 \mathrm{~b}$ & $40.712 \mathrm{c}$ \\
\hline & 126 & $992.86 \mathrm{c}$ & $17960 \mathrm{c}$ & $1419 \mathrm{~b}$ & $13796 \mathrm{c}$ & $1417 \mathrm{~b}$ & $41360 \mathrm{c}$ \\
\hline & 154 & $84143 \mathrm{~b}$ & $17686 \mathrm{c}$ & $1158 \mathrm{ab}$ & $13337 \mathrm{c}$ & $1386 \mathrm{~b}$ & $36300 \mathrm{c}$ \\
\hline
\end{tabular}

1 MV materia verde, MS materia seca, PC proteína cruda, FND fibra neutro detergente, CEN cenizas, ED energía digestible.

a,b,c,d marcan diferencia significativas $(P<0,05)$. 


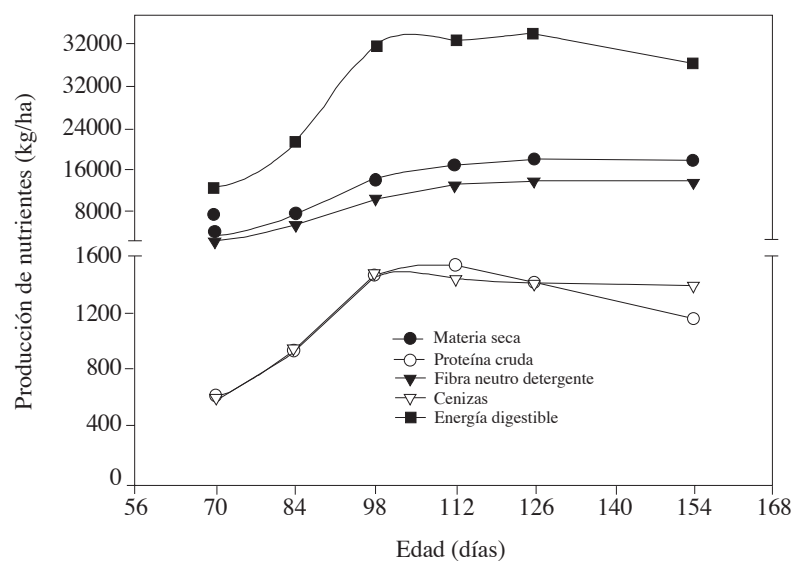

Figura 2. Producción de materia seca y de nutrientes en la planta entera de maíz en diferentes edades de crecimiento. Tres Ríos, Cartago, 2003.

126 días. En hojas y tallos, la mayor acumulación de proteína y energía se produjo a los 98 días y en la mazorca a los 126 días, para luego decrecer lentamente (Figura 2). En términos relativos, a los 126 días, las hojas y las mazorcas retenían cerca del $80 \%$ de la proteína cruda, con una participación similar cada parte de la planta. La fracción restante se encontró en el tallo. A esta edad, las hojas y los tallos reunieron en partes iguales el $60 \%$ de la energía digestible y la mazorca aportó el $40 \%$ restante (Cuadro 3).

Cuadro 3. Distribución porcentual de la materia seca, proteína, fibra y energía en las partes componentes de de la planta Tres Ríos, Cartago, 2003.

\begin{tabular}{lcccccc}
\hline $\begin{array}{c}\text { Parte } \\
\text { planta }\end{array}$ & $\begin{array}{c}\text { Edad } \\
\text { días }\end{array}$ & $\begin{array}{c}\text { MS1 } \\
\text { \%/ } \\
\text { planta }\end{array}$ & $\begin{array}{c}\text { PC1 } \\
\text { \%/ } \\
\text { planta }\end{array}$ & $\begin{array}{c}\text { FND1 } \\
\text { \%/ } \\
\text { planta }\end{array}$ & $\begin{array}{c}\text { CEN1 } \\
\text { \%/ } \\
\text { planta }\end{array}$ & $\begin{array}{c}\text { ED1 } \\
\text { \%/ } \\
\text { planta }\end{array}$ \\
\hline Hoja & 70 & 61,62 & 69,01 & 62,95 & 57,40 & 61,68 \\
& 84 & 53,18 & 68,08 & 53,61 & 58,69 & 53,60 \\
& 98 & 39,08 & 51,21 & 39,84 & 51,56 & 37,83 \\
& 112 & 36,09 & 46,54 & 36,30 & 56,72 & 35,97 \\
& 126 & 31,87 & 41,99 & 32,34 & 55,29 & 29,05 \\
& 154 & 29,37 & 36,91 & 30,75 & 51,58 & 27,50 \\
Tallo & 70 & 38,38 & 30,99 & 37,05 & 42,60 & 38,32 \\
& 84 & 46,82 & 31,92 & 46,39 & 41,31 & 46,40 \\
& 98 & 50,33 & 35,25 & 51,06 & 40,94 & 49,35 \\
& 112 & 38,67 & 24,90 & 37,49 & 30,06 & 35,12 \\
& 126 & 32,85 & 18,33 & 32,54 & 28,85 & 30,83 \\
& 154 & 35,79 & 22,51 & 36,88 & 33,21 & 29,28 \\
Mazorca & 98 & 10,59 & 13,54 & 9,10 & 7,49 & 12,82 \\
& 112 & 25,24 & 28,56 & 26,22 & 13,22 & 28,92 \\
& 126 & 35,27 & 39,68 & 35,12 & 15,87 & 40,11 \\
& 154 & 34,84 & 40,59 & 32,37 & 15,21 & 43,21 \\
\hline
\end{tabular}

1 MS materia seca, PC proteína cruda, FND fibra neutro detergente, CEN cenizas, ED energía digestible.

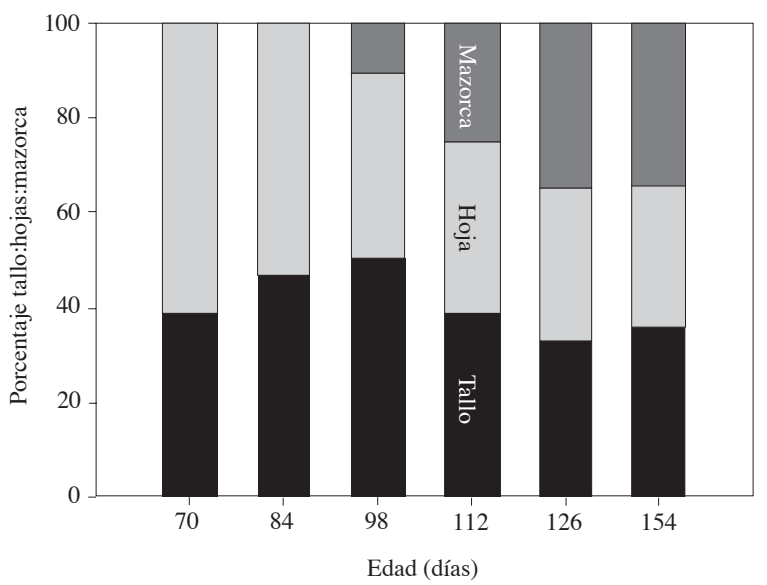

Figura 3. Proporciones de materia seca en tallos, hojas y mazorcas en las diferentes edades de crecimiento. Tres Ríos, Cartago, 2003.

\section{DISCUSIÓN}

Los materiales genéticos híbridos de maíz para la producción de forraje, probados en las fincas lecheras de altura (>1.500 msnm) en los últimos años, mostraron rendimientos inferiores a $30 \mathrm{t} / \mathrm{ha}$ de materia verde, con una humedad superior al $80 \%(<6 \mathrm{t} / \mathrm{ha}$ de materia seca). Ambas características incidieron fuertemente sobre el costo final de cada kilo de ensilado puesto en la boca del animal (Boschini 2002). La segunda característica, pone en riesgo la calidad del ensilaje, tornando el proceso fermentativo láctico a alcohólico (Perry et al. 1967) en el mejor de los casos. En estas condiciones, Yahaya et al. (2002) reportaron que el consumo de carbohidratos solubles en agua es del $82 \%$ al tercer día de ensilado. Los resultados de la presente investigación muestran la capacidad potencial del híbrido 3002 W@ blanco, tanto en rendimiento como en calidad bromatológica. El contenido de materia seca en las hojas (Cuadro 1) muestra que están listas para ensilar a los 112 días desde la siembra; sin embargo, a esta edad aún el tallo conserva $86 \%$ de humedad y la mazorca $88,5 \%$, dando un valor cercano a $15 \%$ de materia seca en la planta total. A cuatro meses de crecimiento, el contenido de proteína fue de $9,2 \%$ y la energía digestible disminuyó a 2,3 mcal $/ \mathrm{kg}$ de materia seca. Un mes antes la calidad fue excelente ( $12 \%$ de proteína cruda y 2,8 $\mathrm{mcal} / \mathrm{kg}$ de energía digestible - Wilkerson et al. 1997), el rendimiento fue bajo (45\%) y la humedad cercana al 90\%. En la Figura 4 se muestra el desarrollo masal de la planta y la evolución de los contenidos de proteína y energía a través del periodo de crecimiento. Se remarca que el dominio de producción empieza a los 112 días, cuando la planta se aproximó a la máxima producción de materia seca, con una considerable pérdida de proteína y energía, llegando a valores cercanos de 


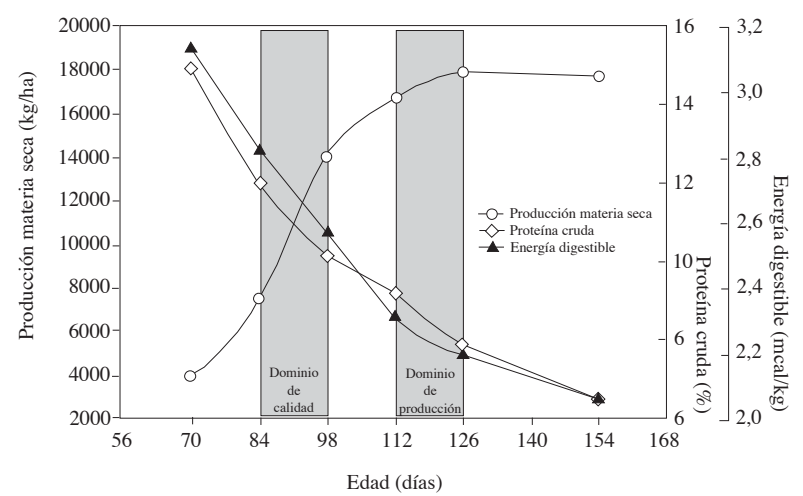

Figura 4. Producción de materia seca y concentración de energía y proteína. Tres Ríos, Cartago, 2003.

mínima concentración de materia seca permitida para lograr con éxito el proceso de ensilaje (Leask y Daynard 1973, Soto y Jahn 1983). En el dominio de calidad se observa que la concentración de proteína y energía fue alta para maíz, así como el contenido de humedad, características propias de un forraje suculento apto únicamente para consumo en fresco (McCaffree y Merrill 1968). El maíz híbrido tuvo ventaja en el rendimiento de materia seca sobre el maíz criollo adaptado a la zona alta de Cartago (17,8 t/ha vs 15,2 t a 126 y 121 días respectivamente) reportado por Amador et al. (2000) y una menor concentración de proteína cruda (2 a 4\%). Los niveles de fibra neutro detergente y las fracciones componentes, fueron concordantes a las reportadas por Elizondo et al. (2001). Los niveles de cenizas (14 a 7\%) se encontraron en el mismo rango, en similares edades de crecimiento.

La extracción parcial o total de elotes para la venta, con el propósito de cubrir parcialmente los gastos del cultivo parece atractivo. Jiménez et al. (2002) trabajaron con el híbrido HS-5G (Cristiani Bukard) reportaron que la extracción del $50 \%$ de elotes permitió recuperar el $89 \%$ de los gastos, a consecuencia de reducir el $24 \%$ del rendimiento de materia seca. Con el híbrido $3002 \mathrm{~W} ®$ la extracción total de elotes representaría una disminución de 25 al 35\% de la producción de materia seca, entre 112 y 126 días de edad, respectivamente, y entre 28 y $40 \%$ de la proteína cruda y de la energía digestible.

\section{CONCLUSIONES}

El híbrido $3002 \mathrm{~W} ®$ blanco (Pionner) mostró un desarrollo vigoroso de 70 a 154 días de crecimiento, con un rendimiento final de 17,9 t/ha. Los contenidos de proteína cruda y energía digestible fueron altos hasta los 84 días (>12\% PC y > 2,8 mcal $/ \mathrm{kg}$ ED) y medios hasta los 112 días (> 9\% PC y $>2,3 \mathrm{mcal} / \mathrm{kg}$ ED). Antes de los 4 meses, el contenido de materia seca fue menor de $15 \%$, recomendado solamente para suministrar a los animales en fresco. Para ensilaje es recomendable esperar hasta los 154 días de crecimiento en altitudes superiores a $1.500 \mathrm{msnm}$ o hasta que alcance un mínimo de $20 \%$ de materia seca.

\section{LITERATURA CITADA}

ALDRICH, S.; LENG, E. 1974. Producción moderna de maíz. Editorial Hemisferio Sur. Argentina. 308 p.

AMADOR, L.; BOSCHINI, C. 2000. Fenología productiva y nutricional de maíz para la producción de forraje. Agronomía Mesoamericana 11(1): 171-177.

AOAC. 2000. Official Methods of Analysis. 17th edition. Association of Official Analytical Chemistry. Washington, DC. Chapter 4: 1-56.

BOSCHINI, C.; AMADOR, L. 2001. Degradabilidad ruminal de la planta de maíz forrajero en diferentes edades de crecimiento. Agronomía Mesoamericana 12:89-93.

BOSCHINI, C. 2002. VII Curso teórico y práctico de ensilaje. Agosto 2002. Estación Experimental "Alfredo Volio Mata”, Universidad de Costa Rica. 165 p.

BOSCHINI, C.; ELIZONDO, J. 2004. Rendimiento de forraje de dos materiales genéticos de maíz sembrados a diferentes distancias de siembra. Revista Agricultura Tropical 34: 87-92.

BRUNO, O.; ROMERO, L.; DIAZ, M.; GAGGIOTTI, M. 1995. Efecto del momento de corte del maíz para ensilaje sobre la producción de leche. INTA. Reporte Técnico. Argentina. $100 \mathrm{p}$.

CUOMO, G.; REDFEARN, D.; BLOUIN, D. 1998. Plant density effects on tropical corn forage mass, morphology, and nutritive value. Agronomy Journal 90:93-96.

ELIZONDO, J.; BOSCHINI, C. 2001. Efecto de la densidad de siembra sobre el rendimiento y la calidad del forraje de maíz. Agronomía Mesoamericana 12(2): 181187.

GOERING, H.K.; VAN SOEST, P.J. 1970. Forage fiber analysis (Apparatus, Reagents, procedures and some Applications). Agricultural Handbook No. ARS-USDA, Washington, DC. $76 \mathrm{p}$.

JIMÉNEZ, C.; PINEDA, L.; LEÓN, B.; MONTENEGRO, A. 2002. Producción de maíz y soya forrajera para ensilaje 
y venta parcial de la cosecha de elotes o chilotes. Agronomía Mesoamericana 13:45-48.

LEASK, W. C; DAYNARD, T.B. 1973. Dry matter yield, in vitro digestibility, percent protein, and moisture of corn stover following grain maturity. Canadian Journal of Plant Science 53:515-522.

McCAFFREE, J.D.; MERRILL, W.G. 1968. High moisture corn for dairy cows in early lactation. Journal of Dairy Science 51:553-560.

PERRY, W.M.; BEESON, W.M.; MOHLER, M.T. 1967. Two types of low-moisture silage fed with three levels of corn and three levels of protein supplement to beef steers. Journal of Animal Science 26:110-114.

SAS. 1985. Statistical Analysis System. SAS User's Guide: Statistics (Version 5 Ed.) SAS Institute Inc. Cary, NC. $373 \mathrm{p}$.

SOTO, P.; JAHN, E. 1983. Época de cosecha y acumulación de materia seca en maíz para ensilaje. Agricultura Técnica. 43(2):133-138.
STEEL, R.; TORRIE, J. 1988. Bioestadística: Principios y Procedimientos. McGraw Hill. México, D.F. 633 p.

TILLEY, J.M.A.; TERRY, R.A. 1963. A two-stage technique for the in vitro digestion of forage crops. Journal of British Grassland Society 18:104-109.

VÁSQUEZ, A. 1982. Estudio detallado de los suelos de la Estación Experimental de Ganado Lechero El Alto. Escuela de Fitotecnia, Facultad de Agronomía, Universidad de Costa Rica. 36 p.

WILKERSON, V.A.; GLENN, B.P.; McLEOD, K.R. 1997. Energy and nitrogen balance in lactating cows fed diets containing dry or high moisture corn in either rolled and ground form. Journal of Dairy Science 80:2487-2496.

YAHAYA, M.S.; KAWAI, M.; TAKAHASHI, J.; MATSUOKA, S. 2002. The effects of different moisture content and ensiling time on silo degradation of structural carbohydrate of Orchardgrass. Asian-Australasian Journal of Animal Science 15:213-217. 